\title{
Epsilon Interactive Virtual User Manual (VUM)
}

\author{
Omar Al Hashimi \\ School of Engineering \\ London South Bank University \\ London, UK \\ alhashio@1sbu.ac.uk
}

\author{
Perry Xiao \\ School of Engineering \\ London South Bank University \\ London, UK \\ xiaop@1sbu.ac.uk
}

\begin{abstract}
Virtual Reality (VR) is an essential technology in today's internet world. $V R$ is capable of delivering an exceptionally competent understanding of a topic, demonstrating a tool, or even conveying an idea through its incredibly realistic environment. Epsilon is a state of the art skin measurement instrument. Initially developed by London South Bank University research group as a research project. Epsilon has now been marketed and utilised in many organisations worldwide. Nevertheless, Epsilon requires thorough on-site training which is costly and time-consuming. Therefore, there is a real need for a Virtual User Manual (VUM) to train clients on how to operate Epsilon and demonstrate all its features and functionalities. This paper presents the development process of a web-based interactive 3D virtual user manual for the Epsilon medical device. The VUM is a step by step interactive 3D guide that will display to users, trainees and clients all of Epsilon's characteristics with the aid of audio and illustrative text. Additionally, it will direct them on how to operate this skin medical instrument efficiently. The interactive VUM will provide users with an extremely realistic ambience presented in 3D content employing The Internet as a delivery method.
\end{abstract}

Keywords-3D modelling, Web3D, Virtual Reality (VR), LMS (Learning Management System), E-Learning, Interactive Learning Environments, Medical Education, Epsilon, 3ds Max, Virtual User Manual (VUM), Virtual Learning Environment (VLE)

\section{INTRODUCTION}

This research paper demonstrates the technology of VR and its importance in delivering a detailed and explicit notion or concept to users via producing realistic atmosphere. The illustration of features for any purchased device is another fundamental aspect of exploiting all the functions of that device or instrument. Virtualisation or the virtual world has always been connected to $3 \mathrm{D}$, as it is the best way to presents and display any $3 \mathrm{D}$ object, picture, movie etc. The term virtualisation defines the separation of a resource or request for a service from the underlying physical delivery of that service. To make virtualisation more effective, usable and beneficial is to add the interactive 3D multimedia applications that can be used with it particularly when the process is deployed online [1]. Moreover, the current internet users are prepared and ready to move from $2 \mathrm{D}$ to $3 \mathrm{D}$ interfaces. In addition to VR, Augmented Reality (AR), for instance, is a very competitive atmosphere for presenting $3 \mathrm{D}$ objects. VR and AR are unlocking a new level of interaction of the users with the specialised applications [2].

The arrival and the wide use of 3-dimensional contents online such as 3D games and 3D movies with the existence of sophisticated machines which have advanced features and great display capabilities have increased the necessity to follow new 3D techs and create a highly advanced and realistic product. This is a suitable time to expand further and enhance the use of those 3D technologies and interactive worlds to other fields, such as e-commerce, elearning, online training, Learning Management System, tourism, and the medical sector. Adding those 3D interactive tools and environments into these new areas has upgraded and greatly improved the user experience and made it more stimulating. Online 3D virtual environments represent real-world characteristics to consumers and allow them to interact with it by using The Internet as a platform. The concept of VR being integrated with social media nowadays is to strengthen its attractiveness further. However, a recent VMware report states that "the potential of 3D/VR technology in everyday applications can be fully exploited. Simply if accompanied by the development of efficient and easy to use methods of creation, management, search and presentation of interactive 3D multimedia content, which could be used by both expert and nonexpert users" [3]. In this regard, London South Bank University's engineering lab has built and developed Epsilon, which is a skin medical instrument. Illustrating this medical instrument in a VR environment would show its functionality as utilised in the real world.

Comparing Epsilon with other skin medical technologies, it has higher sensitivity, higher repeatability, and most importantly, skin measurement results are independent of the external environment. Epsilon is described as a new scanning device for imaging dielectric permittivity $(\varepsilon)$ that is used for a broad array of soft materials, including plant and animal tissues, liquids, fats, gels, waxes, and powders. Its main feature of electronics and signal processing converts the sensor's original nonlinear signals into refined fine-tuned permittivity scale for imaging attributes such as hydration or recording dynamic processes, for instance, textile wetting or the permeation of liquids through membranes [4]. Epsilon is used in more than 70 organisations worldwide, and there are hundreds of people using it [5]. Furthermore, the current user manual of Epsilon is only available as hardcopy and printed documents only. The Epsilon user manual states that "The system consists of a hand-held probe, a parking base, and an in-vitro stand, securely stored in a purpose-designed case" [6].

This paper examines the utilisation of 3D contents online, and the technical approach followed to design and implement the virtual system of Epsilon device and the VUM. In addition, the paper introduces new hardware that can be used to enhance the operation of Epsilon. Finally, it explains a comparative usability study between the current 
Epsilon VUM and the previous one. The Epsilon instrument is shown in the figure below (Fig. 1).

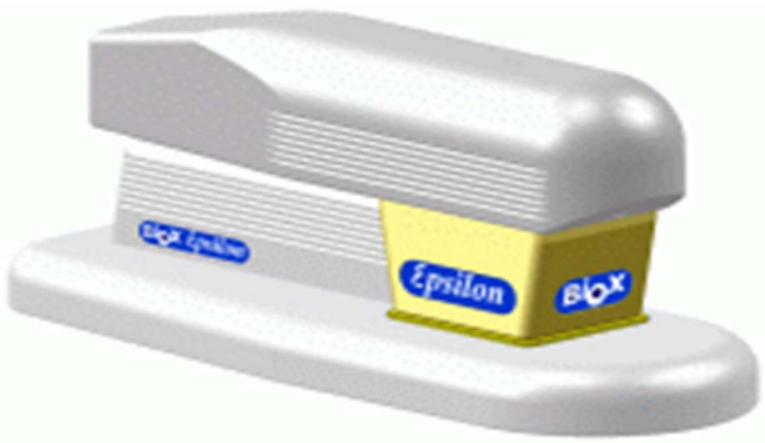

Fig. 1. Epsilon medical instrument

\section{THE UTILISATION OF 3D CONTENTS AND RELATED WORK}

Since the implementation of Web 2.0 era, there has been a noticeable improvement in web applications. Web 2.0 assists and extends into a variety of activities, such as team collaboration, communication, social networking and the huge connection between Internet users and computer. The three-dimensional immersive virtual worlds (3DVW) are one of the essential applications of Web 2.0, which are computer-generated, virtual, online, graphics, multimedia and three- dimensional environments [7]. 3D contents and 3D objects have been around the world of technology since the need for animation and animated content to be displayed in computer systems. The main idea of producing such objects in three dimensions is to show users the reality of these objects. 3D modelling is the key element and the vital work in the domain of VR world [8]. The process of creating $3 \mathrm{D}$ objects can be split into three stages:

a) 3D modelling: the process of building a computer model in 3 dimensions.

b) Layout and animation: the process of adding animation and creating a scene to the object.

c) Rendering: the process of adding other effects to our scenes, such as lights, surface type, positioning the camera and other qualities.

$3 \mathrm{D}$ contents are involved and used immensely in the areas of science, technology, engineering, medical environment, tourism, cosmetic, simulation, e-learning and many more. It has been utilised in the sectors mentioned above jointly with a virtual presentation to stimulate the demonstrated content as is used and experienced in the real world. Some rising technologies will eliminate some of the obstacles in those disciplines. These include computer graphics, AR (Augmented Reality), computational dynamics, and virtual worlds. Recently we have seen some new thoughts coming out in the literature related to the future of education. As has been pointed out by Potkonjak, Gardner, Callaghan, Mattila, Guetl, Petrović, Jovanović (2016), "Technological examples most relevant are distance learning, e-learning, virtual laboratories, virtual reality and virtual worlds, avatars, dynamics-based virtual systems. And, the overall new concept of immersive education that integrates many of these ideas together" [9]. Accordingly, the benefit of adopting VR in the medical and healthcare sectors is to teach and train medical students, trainees and clients on how to use medical devices and instruments and how to conduct some medical tasks. Webbased and online 3D objects used in medical training tools and environments showed to develop the educational process. This web-based virtual medical system of devices for cardiac diagnostic and monitoring functionalities has been created and built to assist in the process of training medical students, qualified health personnel and nonmedical staff to carry out an Electrocardiogram (ECG), an Automatic External Defibrillator (AED) and a blood pressure device.

Those applications guarantee an interactive e-learning experience in the medical field. Also, considering the main objective to emulate real patients, anatomic regions, and clinical tasks and to represent real-life conditions in which this medical tool is built for. These virtual environments allow interaction between users and the system as well as manipulation with very sensitive reactions similar to that of real-life objects. This type of systems will promote learning by practising which makes the whole experience straightforward and enjoyable [10]. Using virtual reality in such projects will help to achieve an extremely immersive experience [11].

\section{BUILDING, AND IMPLEMENTING EPSILON INTERACTIVE} VUM (VIRTUAL USER MANUAL)

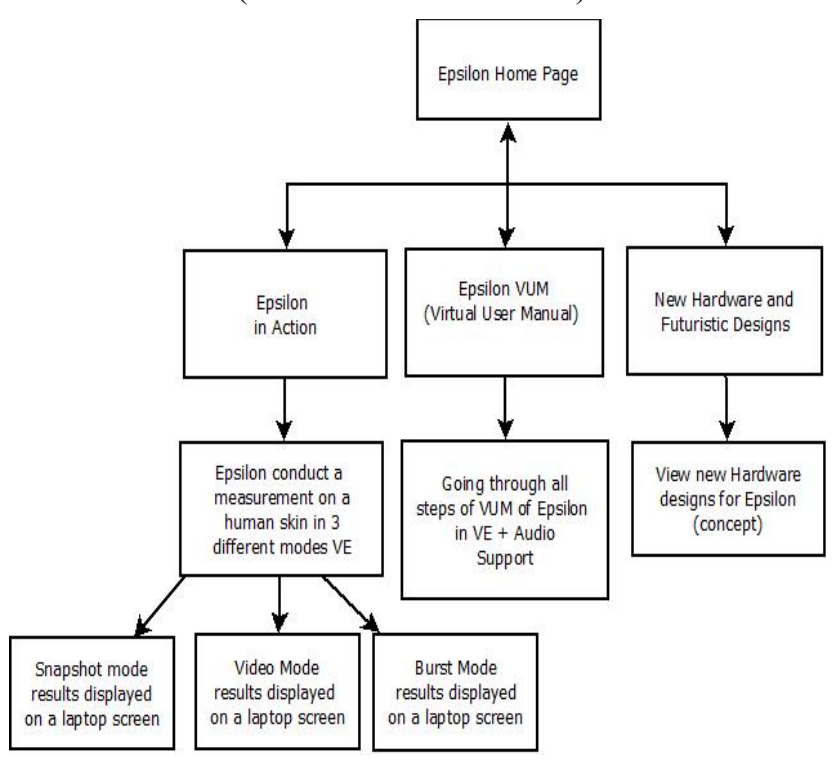

Fig. 2. Flowchart of Epsilon 3D interactive virtual environment

Epsilon's design was not a straightforward process, and the early challenge was selecting the most comprehensive, easy to use and suitable 3D modelling software. However, in Epsilon interactive VUM, the software modelling package used is $3 \mathrm{ds}$ Max. Interactivity between the user (client) and the instrument (Epsilon) has been added using the software Adobe Flash CS6. Other modelling software applications were used at the early stages of the work such as Google Sketchup, Blender and Unity but as a final result of the research was to create, build and develop the 
objects in 3ds Max for its professionalism and a comprehensive set of options features and functions. As mentioned previously, Epsilon is a medical instrument used for skin treatment, and it belongs to the health and medical sector.

The medical field is extremely associated with the use of advanced technologies, such as 3D and VR for demonstration, training, testing and carrying on several medical procedures. Ghanbarzadeh, Ghapanchi, and Blumenstein (2014) point out that "3DVWs (3D Virtual Worlds) have been used in various applications in healthrelated activities, and we identified them in five main categories: education; treatment; simulation; evaluation; lifestyle" [7].

\section{A. Designing and developing Epsilon using 3ds Max modelling package:}

3ds Max is a modelling application that can efficiently and rapidly generate 3D scenes and objects [12]. As in any $3 \mathrm{D}$ modelling process, generating $3 \mathrm{D}$ contents rapidly is an essential element in the design process. Epsilon contains a base part and a probe, on the base part there are few modelling challenges, such as cabling input port and buttons that we need to press to turn the device on or off and to press to start scanning. The probe and the base have been measured with an actual ruler to allow us to create the object as real as possible. Some pictures of the device are considered to capture the accurate proportion of the object, e.g. how big will be the probe compared to a human hand. After the designer evaluates the size and proportion of each object, we use 3D Studio Max to model the instrument. Modelling tools for Epsilon are shown in the figure below:

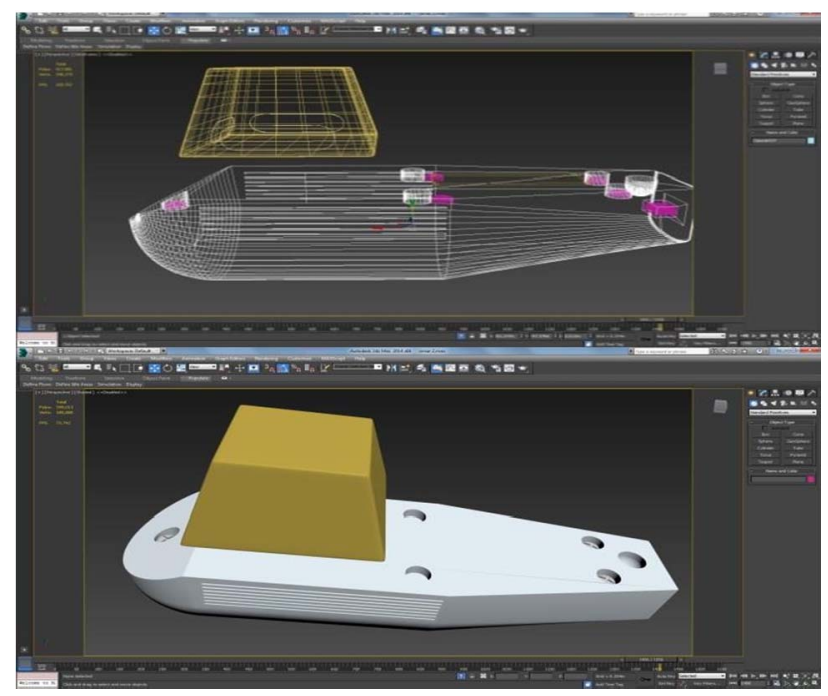

Fig. 3. The modelling of Epsilon in 3ds Max

We can insert different object types from the modelling menu, such as a box, cone, and sphere, tube and cylinder and naming each part. As the process of modelling is going along, the designer needs to make an actual reference to how the newly modelled object is very close to its realistic original copy by placing the original object picture near the modelled one as shown below:

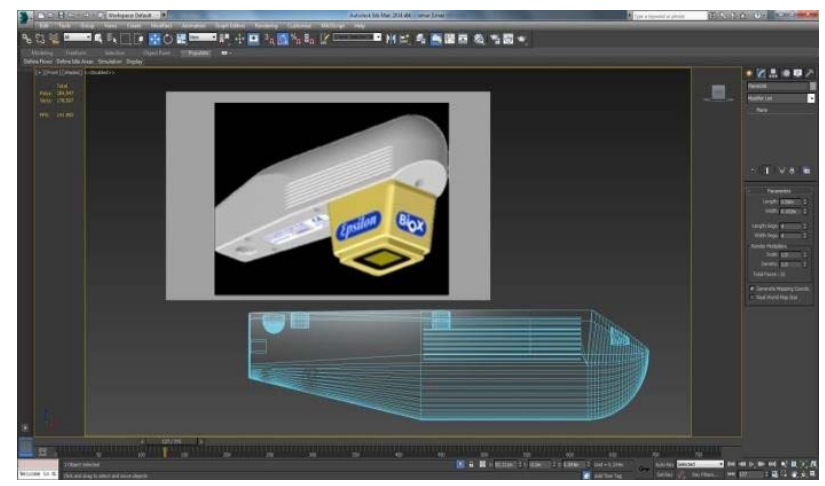

Fig. 4. Epsilon original picture compared to an Epsilon object in 3ds Max

\section{B. Adding material to Epsilon:}

Materials should be considered earlier to fit the modelled object reference if it is realistic enough to meet the client's need. Then, the selected material is carefully studied so that it will not render too slow and too unrealistic. Materials with high glossiness and reflection will consume much of the rendering time. Nevertheless, not adding the best material to the object, the product would appear so unrealistic to the viewers. Vray materials are used in this project, and our third-party renderer is Vray for the whole project, it is one of the industry standard renderers for producing professional-looking images. For the Epsilon Probe and other objects, a semi-gloss material with a whitish material is used. With 0.6 glossiness and a subdivision of 20 , so that it will not acquire much of the rendering time of this object. It is one of the major parts of this design work. HDRI (High Definition Range Imaging) is used for the scene's background. It helps greatly in producing realistic effects of lights and shadows. HDRI is practical to replicate the real world shadows and lighting that will be projected on the objects. Although HDRI takes extra time in rendering, the glossiness and reflectivity of the objects are turned a little bit low, and that will compensate towards the runtime in general.

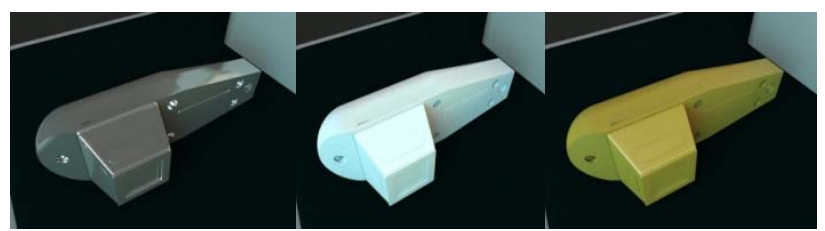

Fig. 5. Adding materials to Epsilon probe in 3ds Max 
After the materials have been selected and added, considering the time and quality of the project, we prepare the objects to be animated. That means all objects that can be grouped will be grouped and linked with each other because if any object that has not been linked together and left out of the other nodes, the keyframes related to that object will disappear and the object will no longer be animated.

\section{Animating Epsilon:}

The animation is a key element and an essential part of any $3 \mathrm{D}$ project design. Nearly a third of the time of the work will be spent on animating the objects modelled. Planning earlier the scenario for each object in the scene how it will interact with the other objects, the motion and the direction of the movement that the specified object will go through in the virtual world. It is a somewhat timeconsuming stage. Unlike character animation, this kind of animation is a bit simpler because it does not need bones and other complex rigging tools. The pivotal points where all motions begin should also be planned before starting the animation process. During the animation period, the timeline is set to 1000 frames and 15 frames per second because this project is specifically designed to be used in an online environment. The frames per second are lowered to accommodate the file size of the images that will be rendered for the online purposes. Using the curve editor to animate objects by editing the curve points, the animated objects can be controlled (speed, timing, and movements).

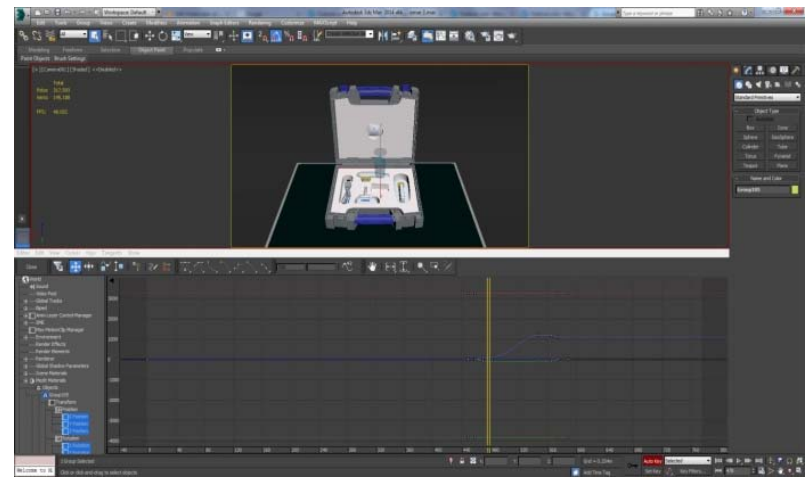

Fig. 6. Using curve editor in 3ds Max

\section{Rendering Epsilon:}

After completing the animation and setting keyframes from a certain point or number into where we want the object to stop at a specific number, then the objects are ready for the final stage of the modelling process which is rendering, where the designer can relax and watch the frames that are being rendered. However, at this stage of the work, it is very important to observe how fast and how long each object will take to finish. Each frame is carefully examined if it is in the right place (number), the objects have the right shadows and lighting. Sometimes objects can be seen floating that are unnoticed during the modelling and animating phase. During rendering, a small window will appear, rendering every square pixel calculating the objects materials, shadows, light reflections etc. In the rendering menu box we can choose the rendered images format, and the images file location, so we can export them into Flash to do all the linking afterwards.

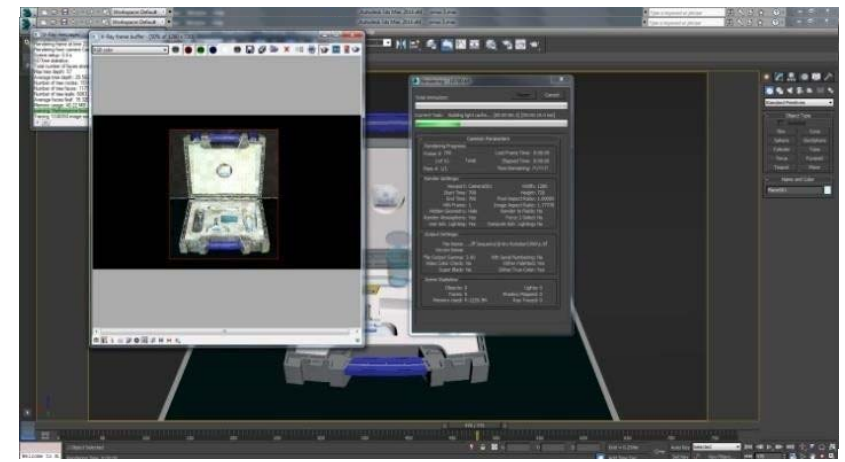

Fig. 7. Rendering window after all keyframes and file setup completed

\section{E. Adding links and Interactivity to Epsilon using Flash CS6:}

To add interactivity to Epsilon, we needed to import all image sequences that we rendered previously using $3 \mathrm{ds}$ max software into a new Flash file:

1) Open flash file with the dimensions of $1920 \times 1080$ HD in scene 1, every one collection of motions can be put together into a scene and enable them to interact to achieve its task.

2) Import images sequence animation into the file library.

3) Create a new symbol and start to drag images into that symbol frame by frame.

4) The image sequence that has been exported to Flash from 3ds Max is being placed on the frames in Flash, each one on a certain frame point to create something similar to the old method of cartoon flip book.

5) When we want to create a button that will stop or end action from occurring, then we only add an ActionScript that contains the word stop(); and attach it to the newly created button.

6) There are many built-in functions for ActionScript already included in Flash under global functions submenu, such as movie clip control functions, timeline control, browse and printing control.

7) For example, under timeline control, we will find common functions, e.g. (play, stop and goto).

8) If any created button that contains a script attached to it needed to be used with its functionality in a different location, then we can only create a new layer in a new scene then copy and paste that button into the new layer, and the new layer will have the exact function as the original button. As well as adding Interactivity functions and buttons to the instrument, voice clips and audio have been added that will play along while displaying the illustrative text once clicked on by the user. 
The above steps can be repeated to all objects that needed to be linked and interacted with the user in a virtual environment via using buttons in Flash.

Epsilon interactive user manual is a fully interactive web-based virtual environment that can present and illustrate all functions, features and a sample skin measurement conducted on a patient in a 3D medical clinic. In (Fig. 8) we can see Epsilon device in operation.

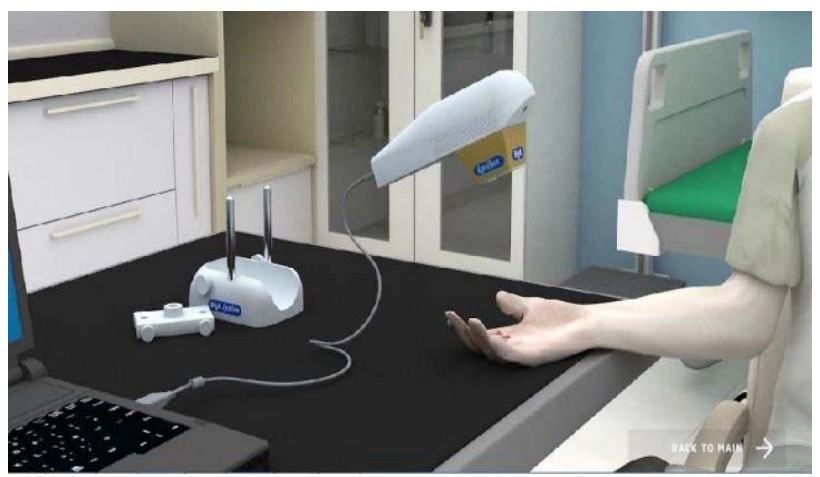

Fig. 8. Epsilon conducting a skin measurement on a patient

After completing the skin measurement, the camera will focus on the laptop's monitor to show the scan results as shown in (Fig. 9).

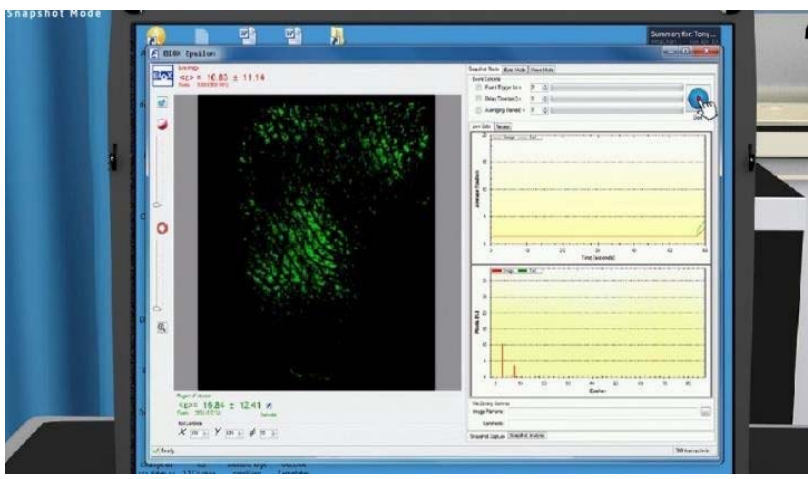

Fig. 9. The measurement result is shown above after clicking on the blue button at the top right corner

In Epsilon device, the user can conduct skin measurements in three different modes, snapshot, burst and video modes. All images and videos of scan results are saved in a folder on the machine's hard drive. All buttons and tabs are designed in an interactive 3D environment and clickable by the user.

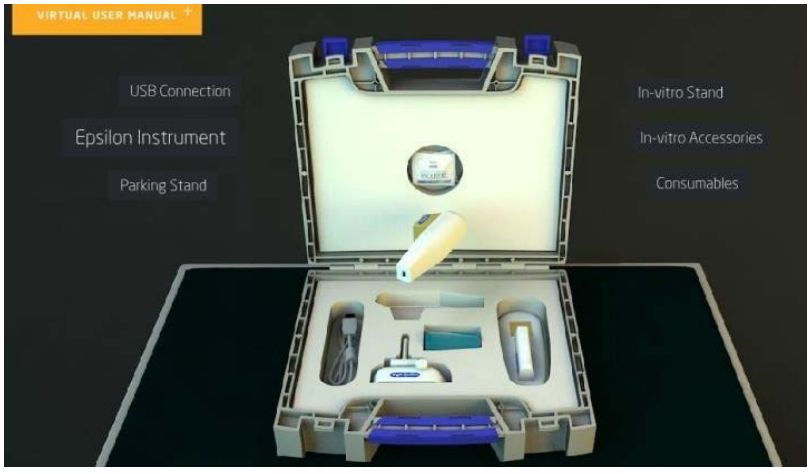

Fig. 10. Epsilon protective case and its parts displayed in a 3D scene

\section{NEW ACCESSORY AND HOLDER DESIGN OF EPSILON}

Soon after completing the main stages of this research study, a fresh design plan came to light, and it contributes positively to the whole measuring process of this project. The notion was to design and create a new accessory (holder) for the skin instrument, in which it is going to add an enormous benefit to the measuring process and will make it effective, quicker and will allow measuring multiple people (patients) at the same time. The holder created has been approved by the BIOX lab at London South Bank University. The new accessory for Epsilon (holder) was carefully planned and meticulously designed to accommodate Epsilon's structure and patient's comfort. The material added to the new accessory was a cloth looking material to imitate the real world object. The new holder is made of gel or silicon material band, and it is wide enough to have a hollow square-shaped holder attached to it that will work as a base for Epsilon to be placed in it. A strap is applied to embrace the Epsilon device firmly in its measuring position.

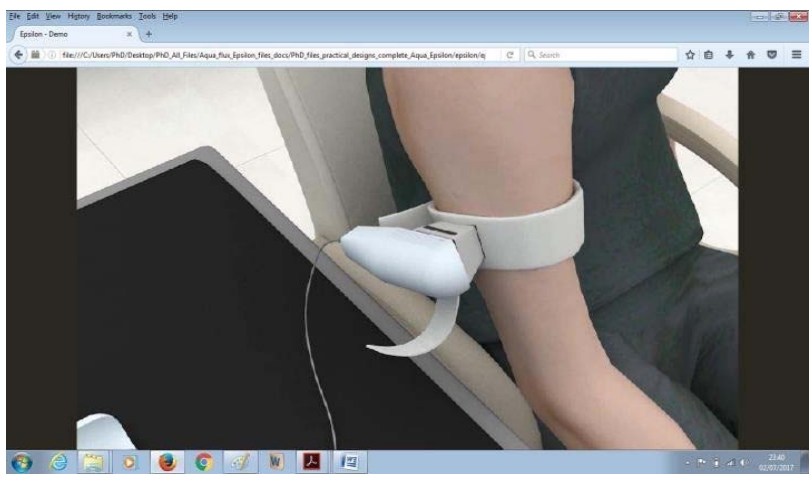

Fig. 11. Epsilon newly designed holder strap in action

All features of the demonstration process performed during the use of the VUM for Epsilon is aided by an audio feature to help guide trainees and users step by step. In addition to the audio feature, there is an illustrative text that will be displayed during each step of the VUM. 


\section{EVALUATING EPSILON}

Epsilon medical instrument currently demonstrated online via this newly invented 3D virtual environment system and the VUM. Previously, a face to face session with each client was required to go through all Epsilon's features and how it operates. The Epsilon VUM system not only is enjoyable to use and easy to access but it covers the illustration of all Epsilon functionalities and characteristics in a virtual ambience.

To evaluate Epsilon, a usability study was carried out in Feb 2018 among 12 users from a non-IT background and what follows are the results:

Eighty-three per cent of the participants found that the service provided online was smooth and error free, whereas $17 \%$ were neutral. Fifty-eight per cent of the users enjoyed their virtual experience, $42 \%$ were neutral. Sixty-four per cent were successful in using the VUM while $36 \%$ were impartial. Fifty-nine per cent were able to control the VUM, whereas 33\% were neutral and $8 \%$ had difficulties. Ninetytwo per cent found that using the support of the audio feature is clear and useful, whereas only $8 \%$ disagreed. Thirty-six per cent thought that the VUM is sufficient and comprehensive, whereas $46 \%$ were neutral and $18 \%$ disagreed.

As is evident from the above results, Epsilon's VUM has a smooth error-free service; users successfully used the VUM, and the audio feature provided was clear and useful. However, there is room to improve the VUM system's as a sufficient tool to teach users how to use the medical device comprehensively.

\section{CONCLUSION}

The goal of this research paper is to present an overview of a VLE (Virtual Learning Environment) which contains $3 \mathrm{D}$ objects. The main contribution to the field is using an interactive virtual environment to demonstrate the process of using a medical device in conducting a human skin measurement for medical purposes with the aid of audio feature. The new Epsilon VUM will replace the old method of illustrating how this medical device work and what are all its features and functionalities via providing each client with an accessible URL after purchasing the instrument. The web-based virtual system is self-explanatory and easy to use as well as the availability of audio support that guides users step by step through the process. Using VR in displaying online 3D contents of the skin measuring tool Epsilon has improved the learning process of how this instrument operates. VR has made all $3 \mathrm{D}$ objects and scenes to be dynamic in response to trainee's action. Allowing the integration of interactivity, dynamic animations, and visual design to challenge and stimulate users. Epsilon VUM system can transform online training into an active, engaging, usable process. Clients can try and test this medical instrument remotely using The Internet as a platform and discover all its features and functions. New hardware and accessories have been presented to illustrate the influence of advertising and convey new ideas realistically to the rest of the world, especially in the era of social media and The Internet used on an enormous scale. The completed work of designing an interactive virtual environment for Epsilon could be simply upgraded into many of today's latest trends in the world of Virtual Reality and technology.

\section{REFERENCES}

[1] Cellary, W. and Walczak, K. (2012). Interactive $3 D$ Multimedia Content. London: Springer London.

[2] Voinea, A., Moldoveanu, F. and Moldoveanu, A. (2017). 3D Model Generation of Human Musculoskeletal System Based on Image Processing. In: 21st International Conference on Control Systems and Computer Science. Bucharest: IEEE, pp.1-3.

[3] VMware Inc, V. (2006). Virtualization Overview White Paper. Palo Alto CA: VMware.

[4] Xiao, P. (2014). Biox Epsilon Model E100: Contact Imaging System. Available at: www.biox.biz/home/brochures.php.

[5] Biox (2016). Biox Science of Measurement. Available at: www.biox.biz/Home/Customers.php.

[6] Biox. (2013). Epsilon E100Manual. London: Biox.

[7] Ghanbarzadeh, R., Ghapanchi, A.H. \& Blumenstein, M. (2014). Application areas of multi-user virtual environments in the healthcare context. Studies in Health Technology and Informatics, 204, pp.38-46.

[8] Shen, W. \& Zeng, W. (2011). Research of VR Modeling Technology Based on VRML and 3DSMAX. , pp.487-490.

[9] Potkonjak, V., Gardner, M., Callaghan, V., Mattila, P., Guetl, C., Petrović, V. and Jovanović, K. (2016). Virtual laboratories for education in science, technology, and engineering: A review. Computers \& Education, 95, pp.309-327.

[10] Violante, M.G. (2015). Politecnico di Torino Porto Institutional Repository Design and implementation of 3D Web-based interactive.

[11] Naber, J., Krupitzer, C. and Becker, C. (2017). Transferring an Interactive Display Service to the Virtual Reality. Mannheim: IEEE, pp.1-4.

[12] Yang, X. et al. (2008). Virtual Reality-Based Robotics Learning System., (September), pp.859-864. 